TRANSACTIONS OF THE

AMERICAN MATHEMATICAL SOCIETY

Volume 280. Number I, November 1983

\title{
TOEPLITZ OPERATORS ON BOUNDED SYMMETRIC DOMAINS \\ BY
}

HARALD UPMEIER

\begin{abstract}
In this paper Jordan algebraic methods are applied to study Toeplitz operators on the Hardy space $H^{2}(S)$ associated with the Shilov boundary $S$ of a bounded symmetric domain $D$ in $C^{n}$ of arbitrary rank. The Jordan triple system $Z \approx \mathbf{C}^{n}$ associated with $D$ is used to determine the relationship between Toeplitz operators and differential operators. Further, it is shown that each Jordan triple idempotent $e \in Z$ induces an irreducible representation (" $e$-symbol") of the $C^{*}$ algebra :T generated by all Toeplitz operators $T_{f}$ with continuous symbol function $f$.
\end{abstract}

0. Introduction. Toeplitz operators on the boundary $\mathbf{T}=\partial \Delta$ of the open unit disc $\Delta \subset \mathbf{C}$ play an important role in function theory of one complex variable (cf. [9, Chapter 7]). In several dimensions Toeplitz operators have been mainly studied for strictly pseudo-convex domains $D \subset \mathrm{C}^{n}[4,13,14,24,28]$, in particular for the Hilbert ball [7], using the relationship with pseudo-differential operators. Another class of domains in $\mathbf{C}^{n}$ generalizing the unit disc is the class of bounded symmetric domains (Cartan domains and exceptional domains), which have a more complicated boundary structure compared to the strictly pseudo-convex case.

The aim of this paper is the study of Toeplitz operators $T_{f}$ with continuous symbol function $f \in \mathcal{E}(S)$ on the Shilov boundary $S$ of a bounded symmetric domain $D$ of arbitrary rank $r$. In the special cases of the Hilbert ball $(r=1)$ and the Lie ball $(r=2)$, the structure of the operators $T_{f}$ and of the Toeplitz $C^{*}$-algebra

$$
\text { T }:=C^{*}\left(T_{f}: f \in \mathcal{e}(S)\right)
$$

generated by these operators is well understood (cf. $[1,2,7])$. The general analysis presented here is based on the Jordan theoretic characterization of bounded symmetric domains. By $[\mathbf{1 6}, \mathbf{2 0}]$ every bounded symmetric domain $D$ can be realized as the open unit ball of a uniquely determined Jordan triple system $Z \approx \mathbf{C}^{n}$ for the so-called spectral norm, and the domains of tube type correspond exactly to the Jordan triple systems defined by complexified formally-real Jordan algebras. The holomorphic and boundary structure of $D$ can be described algebraically in terms of the associated Jordan structures (cf. [20, §6]).

In [27] the Jordan triple system corresponding to $D$ has been used to determine an explicit Peter-Weyl decomposition of the Hardy space $H^{2}(S)$ associated with $S$. Based on the construction of the conical polynomials belonging to the irreducible components of $H^{2}(S)$, our first main result (Theorem 2.1) concerns the relationship

Received by the editors August 25, 1982.

1980 Mathematics Subject Classification. Primary 47B35; Secondary 32M15.

(C)1983 American Mathematical Society $0002-9947 / 83 \$ 1.00+\$ .25$ per page 
between the invariant differential and integral scalar products on these components. As an application it is shown that, similar to the strictly pseudo-convex case, certain "basic" Toeplitz operators can be described by differential operators defined via the Jordan triple product (Theorem 2.11). In $\$ 1$ the fundamental kernel functions (Szegö and Poisson kernel) are characterized in Jordan theoretic terms.

Applying the above results, it is shown in $\S 3$ that each tripotent $e \in Z$ (satisfying the Jordan triple identity $\left\{e e^{*} e\right\}=e$ ) gives rise to an irreducible representation (" $e$-symbol") of the Toeplitz $C^{*}$-algebra $\widetilde{\mathcal{T}}$ on a suitable Hardy space. Actually it can be shown that every irreducible representation of $\sigma$ has this form, which implies that $\sigma$ is a solvable $C^{*}$-algebra (in the sense of [10]) of length $r=\operatorname{rank}(D)$.

1. Toeplitz operators. Let $D$ be a bounded symmetric domain in a complex vector space $Z$ of finite dimension. Without loss of generality we may assume $D$ is circular and contains the origin. The identity component $G$ of the biholomorphic automorphism group $\operatorname{Aut}(D)$ acts on the Shilov boundary $S$ of $D$ in a natural way and the compact linear group

$$
K:=\{g \in G: g(0)=0\}
$$

is transitive on $S$ [20, Theorem 5.3]. Denote by $L^{2}(S)$ the Lebesgue space associated with the unique $K$-invariant probability measure $\mu$ on $S$.

Since $D$ is circular, the Hardy space $H^{2}(S)$ (cf. [17, §4]) can be identified with the closure (in $L^{2}(S)$ ) of the algebra $\mathscr{P}(Z)$ of all polynomials on $Z$. The orthogonal projection $\pi: L^{2}(S) \rightarrow H^{2}(S)$ is called the Szegö projection, being induced by the Szegö kernel $\delta: D \times(D \cup S) \rightarrow \mathbf{C}($ cf. $[17, \S 4])$. Given $f \in L^{\infty}(S)$, the bounded operator $T_{f}$ on $H^{2}(S)$, defined by

$$
T_{f} h:=\pi(f h)
$$

for all $h \in H^{2}(S)$, is called the Toeplitz operator with symbol function $f$. Putting $\bar{f}(s):=\overline{f(s)}$ for all $s \in S$, the definitions imply

$$
T_{f}^{*}=T_{f}^{-}
$$

and

$$
T_{f} T_{g}=T_{f g}
$$

whenever $g \in H^{\infty}(S)$ (the bounded holomorphic functions) and $f \in L^{\infty}(S)$. Here * denotes the Hilbert space adjoint.

Our study of Toeplitz operators will be based on the Jordan theoretic description of bounded symmetric domains in terms of Jordan algebras and Jordan triple systems $[16,20]$. To indicate this relationship, recall that the Lie algebra $g$ of $G$ can be identified with the set of all complete holomorphic vector fields on $D$ [22, Chapter 9]. Holomorphic vector fields will be regarded as differential operators $A=h(z)(\partial / \partial z)$, where $h: D \rightarrow Z$ is holomorphic and $z$ denotes the "coordinate" of $Z$, acting on holomorphic functions $f: D \rightarrow \mathrm{C}$ via

$$
(A \cdot f)(z):=f^{\prime}(z) h(z),
$$


$f^{\prime}(z)$ denoting the derivative of $f$ in $z \in D$. Let $\mathfrak{g}=\mathfrak{f} \oplus \mathfrak{p}$ be the Cartan decomposition with respect to $0 \in D$. By [20, Theorem 4.1] there exists a unique Jordan triple product

$$
Z \times Z \times Z \ni(u, v, w) \mapsto\left\{u v^{*} w\right\} \in Z
$$

such that

$$
\mathfrak{p}=\left\{\left(v-\left\{z v^{*} z\right\}\right)(\partial / \partial z): v \in Z\right\} .
$$

The domain $D$ can be characterized as the open unit ball of the Jordan triple system $Z$ for the so-called spectral norm. It is well known $[16,20]$ that the above construction establishes a 1-1 correspondence between (circular) bounded symmetric domains and the positive definite hermitian Jordan triple systems, called $J B^{*}$-triples in [27]. (By [6] this correspondence can be generalized to domains in complex Banach spaces, thus motivating our terminology.) The group $K$, the largest connected group of Jordan triple automorphisms (cf. [20, §3]) of $Z$, will sometimes be denoted by $K=\operatorname{Aut}(Z)^{0}$. Our first lemma follows from [16, Chapter IV, Theorem 6.2; 17, Remark 4.12 and 19, Theorem 17.3].

Lemma 1.1. For irreducible domains $D$ of rank $r$ and dimension d the Szegö kernel is given by

$$
\varsigma(u, v)=N(u, v)^{-d / r},
$$

where $N$ denotes the generic norm of the associated $J B^{*}$-triple $Z(c f .[19, \S 16])$.

The homogeneous part of bidegree (1.1) of the "sesquipolynomial" mapping $N$ : $Z \times Z \rightarrow C$ is called the generic trace $[19, \S 16]$ and is a $K$-invariant scalar product on $Z$, denoted by (|). By [17, p. 342], the Poisson kernel $\mathscr{P}: D \times S \rightarrow \mathbf{R}$ is given by

$$
\mathcal{P}(z, v):=|\mathcal{S}(z, v)|^{2} / \mathcal{S}(z, z)
$$

for all $(z, v) \in D \times S$. Differentiation and Lemma 1.1 yield

Lemma 1.2. Suppose $D$ is irreducible and $v \in S$. Then the real analytic mapping $\mathscr{P}_{v}$ : $z \mapsto \mathscr{P}(z, v)$ has the derivative

$$
\mathscr{P}_{v}^{\prime}(0) u=(2 d / r) \operatorname{Re}(u \mid v),
$$

where $(\mid)$ denotes the generic trace.

The Shilov boundary of (classical) symmetric domains of rank $\leqslant 2$ is (essentially) a sphere of appropriate dimension. In this special case, Toeplitz operators are closely related to Calderón-Zygmund operators (pseudo-differential operators of order 0) [1, 2, 7]. Using harmonic analysis in $H^{2}(S)$, we will now study relations between Toeplitz operators and certain differential operators for irreducible domains of arbitrary rank.

According to [25] the Hilbert space $H^{2}(S)$, endowed with the $K$-invariant integral scalar product

$$
(f \mid g)_{S}:=\int_{S} \overline{f(s)} g(s) d \mu(s)
$$


admits a Peter-Weyl decomposition (of the dense subspace $\mathscr{P}(Z)$ )

$$
\mathcal{P}(Z)=\sum_{m \in \mathbf{N}_{+}^{r}}^{\oplus} E_{m}
$$

into pairwise inequivalent irreducible unitary $K$-modules $E_{m}$ associated with the "signatures" $m=\left(m_{1}, \ldots, m_{r}\right)$, where

$$
\mathbf{N}_{+}^{r}:=\left\{m=\left(m_{1}, \ldots, m_{r}\right) \in \mathbf{N}^{r}: m_{1} \geqslant \cdots \geqslant m_{r} \geqslant 0\right\} .
$$

On the other hand, the generic trace on $Z$ associates a constant coefficient differential operator $\partial_{p}$ with each polynomial $p \in \mathcal{P}(Z)$ such that

$$
\partial_{l}=v(\partial / \partial z)
$$

where $l=v^{*}$ is defined by $v^{*}(z):=(z \mid v)$ for all $v, z \in Z$. The $K$-invariant scalar product

$$
(p \mid q)_{Z}:=\left(\partial_{p} q\right)(0)
$$

on $\mathscr{P}(Z)$ (cf. $[29,2.1 .4])$ is called the differential scalar product. The decomposition (1.3) is orthogonal for both scalar products and

$$
(p \mid q)_{Z}=C_{m}(p \mid q)_{S}
$$

for all $p, q \in E_{m}$, where $C_{m}>0$ depends only on the signature $m \in \mathbf{N}_{+}^{r}$. Let $C$ : $\mathscr{P}(Z) \rightarrow \mathcal{P}(Z)$ denote the (unbounded) operator in $H^{2}(S)$ defined by

$$
C \mid E_{m}:=C_{m} \cdot \mathrm{id}
$$

for all $m \in \mathbf{N}_{+}^{r}$.

Lemma 1.3. Suppose $f, p, q \in \mathscr{P}(Z)$. Then

$$
\left(T_{f} p \mid q\right)_{Z}=\left(p \mid \partial_{f} q\right)_{Z}
$$

Proof. Using multiplicative properties we may assume $f$ is linear, hence $f=u^{*}$ for some $u \in Z$. Since the subspaces $\mathscr{P}^{k}(Z)$ of all $k$-homogeneous polynomials are mutually orthogonal, we may assume further that $q=\left(v^{*}\right)^{k}$ and $p \in \mathscr{P}^{k-1}(Z)$ for suitable $k \in \mathbf{N}$ and $v \in Z$. In this case,

$$
\begin{aligned}
\left(T_{f} p \mid q\right)_{Z} & =k ! f(v) p(v)=k !(v \mid u) p(v)=k(v \mid u)(k-1) ! p(v) \\
& =k(v \mid u)\left(p \mid\left(v^{*}\right)^{k-1}\right)_{Z}=\left(p \mid \partial_{f} q\right)_{Z} \cdot \quad \text { Q.E.D. }
\end{aligned}
$$

COROLlaRY 1.4. The adjoint (with respect to $H^{2}(S)$ ) of the Toeplitz operator $T_{f}$ for $f \in \mathscr{P}(Z)$ satisfies

$$
T_{f}^{*}=C \partial_{f} C^{-1},
$$

where $C$ is the "diagonal" operator in $H^{2}(S)$ defined in (1.5).

The transformation properties of the Poisson kernel $\mathscr{\rho}$ of $D$ (cf. $[17, \S 4]$ ) imply that for every $g \in G$, with $z:=g(0)$, the image measure $g_{*} \mu$ has the density function $\mathscr{P}_{z}(v):=\mathscr{P}(z, v)$ for all $v \in S$, i.e. $g_{*} \mu=\mathscr{P}_{z} \cdot \mu$. This leads to the following result which will be considerably sharpened in $\$ 2$. 
Proposition 1.5. Let $Z$ be an irreducible $J B^{*}$-triple of rank $r$ and dimension $d$. Then the operator identity

$$
\left\{z v^{*} z\right\} \frac{\partial}{\partial z}=\left(v \frac{\partial}{\partial z}\right)^{*}-\frac{d}{r} T_{l}=C T_{l} C^{-1}-\frac{d}{r} T_{l}
$$

holds on $\mathcal{P}(Z)$ for every $v \in Z$ and $l:=v^{*}$.

Proof. Consider the vector field

$$
A:=\left(v-\left\{z v^{*} z\right\}\right)(\partial / \partial z) \in \mathfrak{g}
$$

and put $g_{t}:=\exp (t A)$ for all $t \in \mathbf{R}$. Then

$$
\left(p \circ g_{t} \mid q \circ g_{t}\right)_{S}=\int_{S} \bar{p} q d\left(\left(g_{t}\right)_{*} \mu\right)=\int_{S} \bar{p} q_{g_{t}(0)} d \mu=\left(p \mid q \mathcal{P}_{g_{t}(0)}\right)_{S}
$$

for all $p, q \in \mathscr{P}(Z)$. Differentiation and Lemma 1.2 yield

$$
(A \cdot p \mid q)_{S}+(p \mid A \cdot q)_{S}=\left(p \mid T_{f} q\right)_{S}
$$

where $f(z):=(2 d / r) \operatorname{Re}(v \mid z)$. Polarization and Corollary 1.4 yield the assertion. Q.E.D.

A bounded symmetric domain $D$ is said to be of tube type if $D$ is holomorphically equivalent to a tube domain over a self-dual homogeneous cone. In terms of the associated $J B^{*}$-triple $Z$, this case is characterized by the existence of a unitary element $e \in Z$ (satisfying $\left\{e e^{*} z\right\}=z$ for all $z \in Z$ ) making $Z$ into a Jordan algebra with unit $e$, product $z \circ w:=\left\{z e^{*} w\right\}$ and involution $z^{*}:=\left\{e z^{*} e\right\}$. The Jordan algebras obtained in this way (called $J B^{*}$-algebras in [27]) are precisely the complexifications of formally-real Jordan algebras [5, Chapter XI].

Toeplitz operators on symmetric domains of tube type have special properties which derive from the existence of the norm function $N \in \mathscr{P}(Z)$ of the corresponding $J B^{*}$-algebra $Z$, normalized by $N(e)=1$ and related to the generic norm of the underlying $J B^{*}$-triple via $N(e-z)=N\left(e, z^{*}\right)$ for all $z \in Z$ (cf. [19, p. 178, Theorem 16.11]). By [27, Lemma 3.8], the norm function satisfies $|N(z)|=1$ and $\left(\partial_{l} N\right)(z)=\overline{l(z)} N(z)$ for all $z \in S$ and every linear form $l$ on $Z$. Applying (1.1) and (1.2), it follows that

$$
T_{N}^{*} T_{N}=\mathrm{id}
$$

and

$$
T_{N}^{*} T_{\partial, N}=T_{l}^{*} .
$$

According to [27, Theorem 2.6], the cokernel of the isometry $T_{N}$ can be characterized as follows:

Proposition 1.6. Let $Z$ be an irreducible $J B^{*}$-algebra of rank $r$ with norm function $N$. Denote by $S^{\prime}:=\{z \in S: N(z)=1\}$ the "reduced Shilov boundary" of the open unit ball $D \subset Z$. Then $\operatorname{ker}\left(T_{N}^{*}\right)$ is the closure of the vector space

$$
\mathcal{H}(Z)=\left\{p \in \mathscr{P}(Z): \partial_{N} p=0\right\}=\sum_{m \in \mathbf{N}_{+}^{r-1}}^{\oplus} E_{m}
$$


of all harmonic polynomials on $Z$. Further, the restriction mapping $\rho: \mathscr{P}(Z) \rightarrow L^{2}\left(S^{\prime}\right)$, defined by $\rho(p):=p \mid S^{\prime}$, induces a Hilbert space isomorphism

$$
\overline{\mathcal{H}(Z)} \approx L^{2}\left(S^{\prime}\right) .
$$

Here we have used the natural identification

$$
\mathbf{N}_{+}^{r-1}=\left\{\left(m_{1}, \ldots, m_{r}\right) \in \mathbf{N}_{+}^{r}: m_{r}=0\right\} .
$$

In view of [27, Theorem 3.7] and (1.8), there exists a Hilbert space tensor product realization

$$
H^{2}(S)=\overline{\mathcal{H}(Z)} \otimes H^{2}(\mathbf{T}),
$$

where $\mathbf{T} \subset \mathbf{C}$ denotes the 1-torus. Obviously,

$$
T_{N}=\mathrm{id} \otimes T_{\xi},
$$

where $\xi$ denotes the identity function on $\mathbf{T}$. Similar tensor product realizations hold for other "basic" Toeplitz operators (cf. [1, 2]):

Proposition 1.7. Let $Z$ be an irreducible JB*-algebra with norm function N. Given $v \in Z$, let $l:=v^{*}$ be the associated linear form. Then there exist tensor product representations

$$
T_{l}=A_{v}^{*} \otimes \mathrm{id}+B_{v}^{*} \otimes T_{\xi}
$$

and

$$
T_{\partial_{\iota} N}=B_{v} \otimes \mathrm{id}+A_{v} \otimes T_{\xi},
$$

where $A_{v}$ and $B_{v}$ are bounded linear operators on $\overline{\mathcal{K}(Z)}$.

Proof. For any $f \in \mathscr{P}(Z), T_{f}^{*}$ leaves $\overline{\mathcal{H}(Z)}$ invariant since $\left[T_{f}^{*}, T_{N}^{*}\right]=\left[T_{N}, T_{f}\right]^{*}$ $=0$. Given $q \in \mathcal{H}(Z)$, Theorem 2.6 of [27] results in a unique representation

$$
\left(\partial_{l} N\right) q=\sum_{k \geqslant 0} N^{k} q_{k}
$$

where $q_{k} \in \mathcal{H}(Z)$ for all $k$. Applying (1.6) and (1.7) yields

$$
T_{l}^{*} q=\sum_{k \geqslant 1} N^{k-1} q_{k} \in \overline{\mathcal{H}(Z)}
$$

which implies $q_{k}=0$ for all $k>1$. It follows that

$$
\left(\partial_{l} N\right) q=B_{v} q+N A_{v} q
$$

for all $q \in \overline{\mathcal{H}(Z)}$ and certain bounded operators $B_{v}$ and $A_{v}$ on $\overline{\mathcal{H}(Z)}$. This implies (1.11). Further, (1.10) follows with (1.6) and (1.7). Q.E.D.

Proposition 1.8. Let $Z$ be an irreducible JB*-algebra. Suppose $u, v \in Z$ and $l:=v^{*}$.Then

$$
\begin{array}{cc}
A_{v}=T_{l}^{*}\left|\overline{\mathcal{H}(Z)}, \quad B_{v}^{*}=T_{\partial_{l} N}^{*}\right| \overline{\mathcal{H}(Z)} . \\
{\left[A_{u}, A_{v}\right]=0=\left[B_{u}, B_{v}\right],} & {\left[A_{u}, B_{v}\right]=\left[A_{v}, B_{u}\right],} \\
{\left[A_{u}, B_{v}^{*}\right]=0=\left[B_{u}, A_{v}^{*}\right],} & {\left[A_{u}, A_{v}^{*}\right]=\left[B_{v}^{*}, B_{u}\right] .}
\end{array}
$$

The following lemma will be used in $\S 2$. 
Lemma 1.9. Let $Z$ be an irreducible $J B^{*}$-algebra of rank $r$ and dimension $d$. Suppose $v \in Z$ and $k \in \mathbf{N}$. Then for every harmonic polynomial $q \in \mathcal{H}(Z)$,

$$
\left\{z v^{*} z\right\} \frac{\partial}{\partial z}\left(N^{k} q\right)=N^{k} q_{0}+\left(k+1-\frac{d}{r}\right) N^{k+1}\left(B_{c}^{*} q\right),
$$

where $q_{0} \in \mathcal{H}(Z)$.

Proof. According to Propositions 1.5 and 1.7,

$$
h:=\left\{z v^{*} z\right\}(\partial / \partial z)\left(N^{k} q\right)=N^{k} q_{0}+N^{k+1} q_{1}
$$

for suitable polynomials $q_{0}, q_{1} \in \mathcal{H}(Z)$. Given $p \in \mathcal{H}(Z)$, it follows from (1.6), Proposition 1.5, (1.10) and (1.11) that

$$
\begin{aligned}
\left(q_{1} \mid p\right)_{S} & =\left(h \mid N^{k+1} p\right)_{S}=\left(N^{k} q \mid \partial_{l}\left(N^{k+1} p\right)\right)_{S}-\frac{d}{r}\left(N^{k+1}\left(B_{v}^{*} q\right) \mid N^{k+1} p\right)_{S} \\
& =(k+1)\left(q \mid p \cdot \partial_{l} N\right)_{S}-\frac{d}{r}\left(B_{v}^{*} q \mid p\right)_{S}=\left(k+1-\frac{d}{r}\right)\left(B_{v}^{*} q \mid p\right)_{S},
\end{aligned}
$$

where $l:=v^{*}$. Since $p$ is arbitrary, the assertion follows. Q.E.D.

2. Toeplitz operators and differential operators. In order to make the relation between Toeplitz operators and differential operators indicated in Corollary 1.4 and Proposition 1.5 more precise, we will show how the eigenvalues $C_{m}$ of the diagonal operator $C$ defined in (1.4) can be computed in a uniform manner from the signature $m=\left(m_{1}, \ldots, m_{r}\right)$ of the $K$-module $E_{m}$ and from certain numerical invariants of the domain $D$.

Let $Z$ be the $J B^{*}$-triple associated with an irreducible bounded symmetric domain $D$ of rank $r$. An element $e \in Z$ is called a tripotent ("triple idempotent") if $\left\{e e^{*} e\right\}=e$. Any frame $\left\{e_{1}, \ldots, e_{r}\right\}$ of minimal orthogonal tripotents $[20, \S 5]$ induces a Peirce decomposition

$$
Z=\sum_{0 \leqslant p \leqslant q \leqslant r}^{\oplus} Z_{p q}
$$

where $Z_{p q}:=\left\{z \in Z: 2\left\{e_{j} e_{j}^{*} z\right\}=\left(\delta_{j p}+\delta_{j q}\right) z\right.$ for $\left.1 \leqslant j \leqslant r\right\}$ and $\delta_{j p}$ denotes the Kronecker symbol. The 3-tuple $(r, s, t)$, defined by $s:=\operatorname{dim} Z_{p q}(1 \leqslant p<q \leqslant r)$, $t:=\operatorname{dim} Z_{o q}(1 \leqslant q \leqslant r)[19, \S 17]$, is called the type of $Z$ or of $D$. Then $t=0$ if and only if $Z$ is a $J B^{*}$-algebra.

For $1 \leqslant k \leqslant r$, the $J B^{*}$-subtriple

$$
Z_{k}:=\sum_{r-k<p \leqslant q \leqslant r}^{\oplus} Z_{p q}
$$

is a $J B^{*}$-algebra with unit element $e_{r+1-k}+\cdots+e_{r}$. Let $N_{k} \in \mathcal{P}\left(Z_{k}\right) \subset \mathscr{P}(Z)$ denote the norm function of $Z_{k}$ (in general, for any subspace $\tilde{Z} \subset Z$, embed $\mathscr{P}(\tilde{Z}) \subset \mathcal{P}(Z)$ via the orthogonal projection onto $\tilde{Z})$. Then it has been shown in [27, Theorem 3.1] (cf. also [15]) that for any signature $m=\left(m_{1}, \ldots, m_{r}\right) \in \mathbf{N}_{+}^{r}$ the polynomial

$$
N_{m}:=N_{1}^{l_{1}} \cdots N_{r}^{l_{r}}
$$


is the conical polynomial (cf. [29,3.3, p. 211]) of $E_{m}$, where $l_{j}:=m_{j}-m_{j+1}$ for $j<r$ and $l_{r}:=m_{r}$. Define $L:=\{g \in K: g(e)=e\}$ for $e:=e_{1}+\cdots+e_{r} \in S$. Then

$$
P_{m}:=\int_{L}\left(N_{m} \circ g\right) d g \in E_{m}
$$

is the unique $L$-invariant spherical polynomial with $P_{m}(e)=1$. It is well known (cf. [26, p. 448]) that

$$
\left(P_{m} \mid P_{m}\right)_{S}=\left(\operatorname{dim} E_{m}\right)^{-1} \text {. }
$$

Theorem 2.1. Let $Z$ be an irreducible $J B^{*}$-triple of type $(r, s, t)$. Let $S$ denote the Shilov boundary of the open unit ball $D \subset Z$. Given any signature $m=\left(m_{1}, \ldots, m_{r}\right) \in$ $\mathbf{N}_{+}^{r}$, the differential and integral scalar products are related for $p, q \in E_{m}$ as follows:

$$
\frac{(p \mid q)_{Z}}{(p \mid q)_{S}}=C_{m}=\prod_{j=1}^{r} \frac{\left(m_{j}+\frac{1}{2}(r-j)+t\right) !}{\left(\frac{1}{2}(r-j)+t\right) !} .
$$

For the proof we assume, up to Lemma 2.6 , that $Z$ is a $J B^{*}$-algebra.

Lemma 2.2. Suppose $m, n \in \mathbf{N}_{+}^{r-1} \subset \mathbf{N}_{+}^{r}$ and $\left(B_{v}^{*} E_{n} \mid E_{m}\right)_{S} \neq 0$ for some $v \in Z$. Then

$$
C_{\left(m_{1}+k+1, \ldots m_{r-1}+k+1, k+1\right)}=(k+1) C_{\left(n_{1}+k \ldots, n_{r},+k, k\right)}
$$

for all $k \in \mathbf{N}$.

Proof. Given $q \in E_{n} \subset \mathcal{H}(Z)$, Proposition 1.5 and Lemma 1.9 imply

$$
C N^{k+1}\left(B_{v}^{*} q\right)=(k+1) C_{\left(n_{1}+k, \ldots, n_{r},+k, k\right)} N^{k+1}\left(B_{v}^{*} q\right) \text {. }
$$

Since $\left(B_{v}^{*} q \mid E_{m}\right)_{S} \neq 0$ for some $q$, the assertion follows. Q.E.D.

Lemma 2.3. Suppose $m \in \mathbf{N}_{+}^{r-1}$. Then $\left(B_{v}^{*} E_{n} \mid E_{m}\right)_{S} \neq 0$ for $n:=\left(m_{1}+1, \ldots\right.$, $\left.m_{r-1}+1,0\right)$ and $v:=e_{1}$.

Proof. Put $l:=v^{*}$. Then $N_{r-1}$ and $\partial_{l} N$ coincide on $Z_{r-1}$. Further, $A v=0$ for all vector fields $A$ in the maximal nilpotent subalgebra $n_{+}$of $\mathfrak{f}^{\mathbf{C}}$ spanned by all positive compact root spaces. This is clear if $A \in \mathfrak{f}_{p q}^{\mathrm{C}}$ and $1 \leqslant p<q \leqslant r$ (cf. [27, (1.8)]), and it follows as in the proof of [27, Lemma 3.4] if $A \in \mathfrak{f}_{\alpha}^{C}$ and $\alpha \mid \mathfrak{h}_{-1}=0$; now apply [27, Theorem 1.7]. Therefore $\partial_{l} N$ is $\mathrm{n}_{+}$-invariant and hence can be written as a polynomial in $N_{1}, \ldots, N_{r-1}$ [27, Theorem 3.1]. It follows that $\partial_{l} N=N_{r-1}$ on $Z$. Since $N_{n}=N_{m} \cdot N_{r-1}$ by construction, (1.11) implies

$$
\left(B_{v}^{*} N_{n} \mid N_{m}\right)_{S}=\left(N_{n} \mid \partial_{l} N \cdot N_{m}\right)_{S}=\left(N_{n} \mid N_{n}\right)_{S}>0 \text {. Q.E.D. }
$$

Corollary 2.4. Suppose $n \in \mathbf{N}_{+}^{r-1}$ and $n_{r-1}>0$. Then

$$
C_{\left(n_{1}+k \ldots, n_{r-1}+k, k+1\right)}=(k+1) C_{\left(n_{1}+k, \ldots, n_{r-1}+k, k\right)}
$$

for all $k \in \mathbf{N}$. Hence (2.5) is true for all signatures in $\mathbf{N}_{+}^{r}$ if it holds for all signatures in $\mathbf{N}_{+}^{r-1}$.

Let $B(a, b):=\Gamma(a) \Gamma(b) / \Gamma(a+b)$ denote the Beta-function. 
Lemma 2.5. Denote by $N_{m}$ and $P_{m}$ the conical and spherical polynomials, resp., associated with $m \in \mathbf{N}_{+}^{r}$. Then

$$
\frac{\left(P_{m} \mid P_{m}\right)_{S}}{\left(N_{m} \mid N_{m}\right)_{S}}=\prod_{1 \leqslant p<q \leqslant r} \frac{B\left(m_{p}-m_{q}, \frac{s}{2}(q+1-p)\right)}{B\left(m_{p}-m_{q}, \frac{s}{2}(q-p)\right)} .
$$

Proof. By [25] the $K$-module $E_{m}$ has the highest weight

$$
\mu=-\sum_{k=1}^{r} m_{k} \gamma_{k}
$$

where $\gamma_{1}, \ldots, \gamma_{r}$ denote the Harish-Chandra strongly orthogonal noncompact roots of $\mathfrak{f}^{\mathbf{C}}$ with respect to a Cartan subalgebra $\mathfrak{h}^{\mathbf{C}}$ containing the vector fields $\left\{e_{k} e_{k}^{*} z\right\}(\partial / \partial z)$ for $1 \leqslant k \leqslant r$. Let $\mathfrak{h}^{\mathbf{C}}=\mathfrak{h}_{1}^{\mathbf{C}} \oplus \mathfrak{h}_{-1}^{\mathbf{C}}$ be the canonical decomposition with respect to $e \in S$ (cf. [27, Lemma 1.2]). Then the nonzero restrictions $\lambda=\alpha \mid \mathfrak{h}_{-1}^{\mathrm{C}}$ of positive compact roots $\alpha$ have the form $\lambda=\left(\gamma_{q}-\gamma_{p}\right) / 2$ for $1 \leqslant p<q \leqslant r$, occurring with common multiplicity $m(\lambda)=s$ (cf. [25]). It follows that

$$
\rho:=\frac{1}{2} \sum_{\lambda=0} m(\lambda) \lambda=\frac{s}{2} \sum_{k=1}^{r}\left(k-\frac{r+1}{2}\right) \gamma_{k} .
$$

Choose a $K$-invariant hermitian scalar product $(\mid)$ on $\mathfrak{f}^{\mathbf{C}}$ such that $i z(\partial / \partial z)$ is orthogonal to the derived ideal $\mathfrak{H}^{\prime}$ and $\left(\gamma_{p} \mid \gamma_{q}\right)=2 \delta_{p q}$ for $1 \leqslant p, q \leqslant r$. The dual group of the compact semisimple Lie group $K^{\prime}$ generated by $\mathfrak{f}^{\prime}$ has an Iwasawa decomposition. Therefore the quotient $\left(P_{m} \mid P_{m}\right)_{S} /\left(N_{m} \mid N_{m}\right)_{S}$ can be expressed as an integral (cf. [29, p. 211, proof of Theorem 3.3.1.1]). Evaluating this integral as in [29, 9.1.6, p. 325] yields

$$
\left(P_{m} \mid P_{m}\right)_{S} /\left(N_{m} \mid N_{m}\right)_{S}=I(\mu+\rho) / I(\rho),
$$

where for any linear form $\beta$ on $\mathfrak{h}_{-1}^{\mathbf{C}}$ we put

$$
I(\beta):=\prod_{\lambda>0} B\left(\frac{s}{2}, \frac{(\beta \mid \lambda)}{(\lambda \mid \lambda)}\right) .
$$

Now the result follows by direct computation. Q.E.D.

Lemma 2.6. Suppose $m \in \mathbf{N}_{+}^{r}$. Then

$$
\operatorname{dim} E_{m}=\prod_{1 \leqslant p<q \leqslant r} \frac{m_{p}-m_{q}+\frac{s}{2}(q-p)}{\frac{s}{2}(q-p)} \frac{B\left(m_{p}-m_{q}, \frac{s}{2}(q-p-1)+1\right)}{B\left(m_{p}-m_{q}, \frac{s}{2}(q+1-p)\right)}
$$

and

$$
\frac{1}{\left(N_{m} \mid N_{m}\right)_{S}}=\prod_{1 \leqslant p<q \leqslant r} \frac{B\left(m_{p}-m_{q}, \frac{s}{2}(q-p-1)+1\right)}{B\left(m_{p}-m_{q}, \frac{s}{2}(q-p)+1\right)} .
$$

Proof. For the special cases $r \leqslant 2$ or $s=1$, (2.6) follows easily by inspection and from Weyl's dimension formula [29, Theorem 2.4.1.6]. Hence we may assume $r \geqslant 3$ and $s \geqslant 2$. Then $s$ is even and the positive roots $\alpha$ not vanishing on $\mathfrak{h}_{-1}^{\mathrm{c}}$ have the form

$$
\alpha=\left(\gamma_{q}-\gamma_{p}\right) / 2 \pm \beta_{p q}^{j}
$$


where $1 \leqslant p<q \leqslant r, 1 \leqslant j \leqslant s / 2$ and $\beta_{p q}^{j}$ are linear forms on $\mathfrak{h}^{\mathbf{C}}$ vanishing on $\mathfrak{h}_{-1}^{\mathrm{C}}$ (cf. [25]). Defining $\rho_{0}$ as in [25], it follows from [25, Lemma 12] that

$$
\left\{ \pm\left(\rho_{0} \mid \beta_{p q}^{j}\right): 1 \leqslant j \leqslant s / 2\right\}=\{ \pm(j-1): 1 \leqslant j \leqslant s / 2\} .
$$

Another application of Weyl's formula gives (2.6). Combining (2.6), Lemma 2.5 and (2.4) yields (2.7). Q.E.D.

Proof of Theorem 2.1. We first prove Theorem 2.1 for irreducible $J B^{*}$-algebras $Z$ by induction on the rank $r$ of $Z$. If $r=1$, then $Z=\mathbf{C}$ and (2.5) is clear. If $r=2$, then $Z \approx \mathbf{C}^{n}$ is a "spin factor" of type $(2, n-2,0)$. In this case the assertion (2.5) for signatures $\left(m_{1}, 0\right)$ follows from straightforward calculations (cf. [2]). By Lemma $2.4,(2.5)$ is true for all signatures $m \in \mathbf{N}_{2}^{+}$.

Now assume $Z$ is of type $(r, s, 0)$ with $r \geqslant 3$. Then $\tilde{Z}:=Z_{r-1}$, defined in $(2.1)$, is an irreducible $J B^{*}$-algebra of type $(r-1, s, 0)$. By Lemma 2.4 it suffices to prove (2.5) for signatures $m=\left(m_{1}, \ldots, m_{r-1}, 0\right) \in \mathbf{N}_{+}^{r-1}$. Then, by construction, $N_{m} \in$ $\mathscr{P}(\tilde{Z}) \subset \mathscr{P}(Z)$. Since the generic norms of $Z$ and $\tilde{Z}$ agree on $\tilde{Z}$, it follows that $\left(N_{m} \mid N_{m}\right)_{Z}=\left(N_{m} \mid N_{m}\right)_{\tilde{Z}}$. Since the irreducible $\operatorname{Aut}(\tilde{Z})^{0}$-module generated by $N_{m}$ has signature $\left(m_{1}, \ldots, m_{r-1}\right)$, we can apply the induction hypothesis for $\tilde{Z}$ and (2.7) to conclude that (2.5) holds for $Z$.

Now assume $Z$ is an irreducible $J B^{*}$-triple of type $(r, s, t)$. Then $\tilde{Z}:=Z_{r}$, defined in (2.1), is an irreducible $J B^{*}$-algebra of type $(r, s, 0)$. Further, the group $L$ (cf. (2.3)) commutes with the Peirce projection onto $\tilde{Z}$. By (2.3) the spherical polynomial $P_{m}$ associated with the signature $m \in \mathbf{N}_{+}^{r}$ lies in $\mathcal{P}(\tilde{Z})$, whence

$$
\left(P_{m} \mid P_{m}\right)_{Z}=\left(P_{m} \mid P_{m}\right)_{\bar{Z}} \text {. }
$$

According to (2.4) the integral scalar products are related to the dimensions of the corresponding $K$-module $E_{m} \subset \mathscr{P}(Z)$ and $\operatorname{Aut}(\tilde{Z})^{0}$-module $\tilde{E}_{m} \subset \mathscr{P}(\tilde{Z})$. Therefore the assertion follows from the first part of the proof, applied to $\tilde{Z}$, and from the following lemma. Q.E.D.

Lemma 2.7. Suppose $Z$ is an irreducible $J B^{*}$-triple of type $(r, s, t)$. Then

$$
\frac{\operatorname{dim} E_{m}}{\operatorname{dim} \tilde{E}_{m}}=\prod_{j=1}^{r} \frac{B\left(m_{j}, \frac{s}{2}(r-j)+1\right)}{B\left(m_{j}, \frac{s}{2}(r-j)+t+1\right)} .
$$

Proof. Denote by $F_{\left(n_{1} \ldots, n_{k}\right)}$ the irreducible $\operatorname{GL}(k, \mathrm{C})$-representation with signature $n_{1} \geqslant \cdots \geqslant n_{k} \geqslant 0$. The classical irreducible $J B^{*}$-triples $Z$ with $t>0$ are of type $(r, 2, t)$ or $(r, 4,2)$. In these cases we have, respectively,

$$
\begin{aligned}
& E_{m} \approx F_{\left(m_{1}, \ldots, m_{r}\right)} \otimes F_{\left(m_{1}, \ldots m_{r}, 0, \ldots, 0\right)} \quad(t \text { zeros }), \\
& \tilde{E}_{m} \approx F_{\left(m_{1}, \ldots, m_{r}\right)} \otimes F_{\left(m_{1}, \ldots, m_{r}\right)}, \quad \text { or } \\
& E_{m} \approx F_{\left(m_{1}, m_{1}, \ldots, m_{r}, m_{r}, 0\right)}, \quad \tilde{E}_{m} \approx F_{\left(m_{1}, m_{1}, \ldots, m_{r}, m_{r}\right)} .
\end{aligned}
$$

In both cases Lemma 2.7 follows from [12, (1.2.10)]. For the exceptional domain of type $(2,6,4)$, apply Weyl's dimension formula to $K \approx \operatorname{Spin}(10) \cdot T$. Q.E.D.

We now apply Theorem 2.1 to analyze the fine structure of certain Toeplitz operators. 
Lemma 2.8. Suppose $Z$ is an irreducible $J B^{*}$-algebra, $v \in Z$ and $m \in \mathbf{N}_{+}^{r-1}$. Then $B_{v}\left(E_{m}\right) \subset E_{n}$, where $n:=\left(m_{1}+1, \ldots, m_{r-1}+1,0\right)$.

Proof. Suppose $n \in \mathbf{N}_{+}^{r-1}$ satisfies $\left(B_{v}\left(E_{m}\right) \mid E_{n}\right)_{S} \neq 0$. By Lemma 2.4 it follows that

$$
C_{\left(m_{1}+k+1 \ldots m_{r-1}+k+1, k+1\right)}=(k+1) C_{\left(n_{1}+k \ldots, n_{r-1}+k, k\right)}
$$

for all $k \in \mathbf{N}$. By Theorem 2.1 (applied to $k$ and $k+1$ ), this implies

$$
\prod_{j=1}^{r}\left(m_{j}+k+1+\frac{s}{2}(r-j)\right)=\prod_{j=1}^{r}\left(n_{j}+k+\frac{s}{2}(r-j)\right) \text {, }
$$

whence $m_{j}+1=n_{j}$ for all $j<r$. Q.E.D.

Given a signature $m=\left(m_{1}, \ldots, m_{r}\right) \in \mathbf{N}_{+}^{r}$, define, for $1 \leqslant j \leqslant r$,

$$
m \pm \varepsilon_{j}:=\left(m_{1}, \ldots, m_{j-1}, m_{j} \pm 1, m_{j+1}, \ldots, m_{r}\right)
$$

and put $E_{m \pm \varepsilon_{j}}:=\{0\}$ if $m \pm \varepsilon_{j}$ is not a signature.

Lemma 2.9. Suppose $Z$ is an irreducible $J B^{*}$-triple and $m \in \mathbf{N}_{+}^{r}$. Then the conical polynomial $N_{m}$ satisfies

$$
\left(v \frac{\partial}{\partial z}\right) N_{m} \in \sum_{k<j \leqslant r}^{\oplus} E_{m-\varepsilon}
$$

for all $v \in \sum_{0 \leqslant p \leqslant q \leqslant r-k}^{\oplus} Z_{p q}$ and $0 \leqslant k<r$.

Proof. We may assume $v \in Z_{p q}$ for $0 \leqslant p \leqslant q \leqslant r-k$. Further, we may assume $Z$ is a $J B^{*}$-algebra since $v(\partial / \partial z) N_{m}=0$ if $p=0$. The proof is by induction on $r$, the case $r=1$ being trivial. If $r \geqslant 2$, then $Z_{r-1}$, defined in (2.1), is an irreducible $J B^{*}$-algebra of rank $r-1$. Now $N_{m}=N_{n} \cdot N^{m_{r}}$, where $n:=\left(m_{1}-m_{r}, \ldots, m_{r-1}-\right.$ $\left.m_{r}, 0\right)$. For $l:=v^{*}$, the product rule implies

$$
\left(v \frac{\partial}{\partial z}\right) N_{m}=N^{m_{r}}\left(v \frac{\partial}{\partial z} N_{n}\right)+m_{r} N_{n} N^{m_{r}-1} \partial_{l} N
$$

To treat the first summand, observe that $(v(\partial / \partial z)) N_{n}=0$ if $p=1$. Hence we may assume $0 \neq v \in Z_{r-1}$ and, therefore, $0 \leqslant k<r-1$. The induction hypothesis applied to $Z_{r-1}$ yields

$$
\left(v \frac{\partial}{\partial z}\right) N_{n} \in \sum_{k<j<r}^{\oplus} E_{n-\varepsilon_{j}} .
$$

To treat the second summand, we may assume $m_{r}=1$. If $p<q \leqslant r-k$, then $A:=\left\{v e_{q}^{*} z\right\}(\partial / \partial z) \in \mathrm{n}_{+}$by [27, Theorem 1.7] and a straightforward computation shows

$$
w:=\exp (A)\left(e_{q}\right)=e_{q}+v / 2+a e_{p}
$$

for some $a \in \mathbf{C}$. Since $N$ is $\mathrm{n}_{+}$-invariant, it follows that

$$
\left(w \frac{\partial}{\partial z} N\right)(\exp (A) z)=\left(e_{q} \frac{\partial}{\partial z} N\right)(z)
$$


Hence we may assume $p=q$ and $v=e_{q}$ for $1 \leqslant q \leqslant r-k$. Since $N_{n} \in \mathcal{H}(Z)$, it follows that $\partial_{l} N \cdot N_{n}=B_{v}\left(N_{n}\right)+N \cdot A_{v}\left(N_{n}\right)$. By Lemma $2.8 B_{v}\left(N_{n}\right) \in$ $E_{\left(m_{1}, \ldots, m_{r-1}, 0\right)}$. On the other hand, if $q \geqslant 2$, the induction hypothesis, applied to $v \in Z_{r-1}$, together with Proposition 1.8 and Corollary 1.4 imply

$$
A_{v}\left(N_{n}\right) \in \sum_{k<j<r}^{\oplus} E_{n-\varepsilon_{j}} .
$$

If $q=1$, then $\partial_{l} N=N_{r-1}$ implies $A_{v}\left(N_{n}\right)=0$. Q.E.D.

In view of Corollary 1.4 and Proposition 1.5, Lemma 2.9 has the following consequence:

Corollary 2.10. Suppose $v \in Z$. Then

$$
\left(v \frac{\partial}{\partial z}\right) E_{m} \subset \sum_{1 \leqslant j \leqslant r}^{\oplus} E_{m-\varepsilon}
$$

and

$$
\left\{z v^{*} z\right\} \frac{\partial}{\partial z} E_{m} \subset \sum_{1 \leqslant j \leqslant r}^{\oplus} E_{m+\varepsilon},
$$

Combining Corollary 1.4, Proposition 1.5, Theorem 2.1 and Corollary 2.10 enables us to describe the relationship between "basic" Toeplitz operators and differential operators in an explicit form:

TheOREM 2.11. Suppose $Z$ is an irreducible $J B^{*}$-triple of type $(r, s, t)$. For $v \in Z$ put $l:=v^{*}$. Then

$$
T_{l}^{*} p=\sum_{j=1}^{r}\left(m_{j}+\frac{s}{2}(r-j)+t\right)^{-1}\left(v \frac{\partial}{\partial z} p\right)_{m-\varepsilon_{l}}
$$

and

$$
T_{l} p=\sum_{j=1}^{r}\left(m_{j}-\frac{s}{2}(j-1)\right)^{-1}\left(\left\{z v^{*} z\right\} \frac{\partial}{\partial z} p\right)_{m+\varepsilon_{l}}
$$

for all $p \in E_{m}$ and $m \in \mathbf{N}_{+}^{r}$, the subscript $m \pm \varepsilon_{j}$ denoting the respective Peter-Weyl component.

COROLlaRY 2.12. The commutator $\left[T_{l}^{*}, T_{l}\right]$ is a "diagonal" operator, i.e. $\left[T_{l}^{*}, T_{l}\right] E_{m}$ $\subset E_{m}$ for all signatures $m \in \mathbf{N}_{+}^{r}$.

Proof. Suppose $1 \leqslant j, k \leqslant r$ and $j \neq k$. Then

$$
v \frac{\partial}{\partial z}\left(T_{l} p\right)_{m+\varepsilon_{k}}-T_{l}\left(v \frac{\partial}{\partial z} p\right)_{m-\varepsilon_{j}}
$$

has vanishing $\left(m+\varepsilon_{k}-\varepsilon_{j}\right)$-component, if $m \in \mathbf{N}_{+}^{r}$ and $p \in E_{m}$, since $\left[v(\partial / \partial z), T_{l}\right]$ $=l(v) \cdot$ id is a diagonal operator. Now apply Theorem 2.11. Q.E.D.

3. The Toeplitz $C^{*}$-algebra. The mapping $f \mapsto T_{f}$ from $L^{\infty}(S)$ into the $C^{*}$-algebra $\mathcal{L}(H)$ of all bounded linear operators on the Hilbert space $H:=H^{2}(S)$ is by definition (completely) positive but not a homomorphism. In particular, Toeplitz 
operators are in general not normal and do not necessarily commute. The appropriate object to study Toeplitz operators is therefore the $C^{*}$-algebra $C^{*}\left(T_{f}: f \in \Sigma\right)$ generated by all Toeplitz operators $T_{f}$ with $f$ belonging to a certain "symbol algebra" $\Sigma \subset L^{\infty}(S)$. For $\Sigma=L^{\infty}(S)$, the structure of the corresponding $C^{*}$-algebra is unknown even in the simplest case of the unit circle $S \subset \mathbf{C}$ (cf. [9, §7]). For continuous symbols $f \in \mathcal{C}(S)$ however, there exists a satisfying structure theory for the Toeplitz $C^{*}$-algebra $\mathcal{T}:=C^{*}\left(T_{f}: f \in \mathcal{C}(S)\right)$ associated with the Shilov boundary $S$ of a bounded symmetric domain $D$ of arbitrary rank. The following results concerning the structure of $\mathcal{T}$ generalize results of $\mathrm{C}$. Berger, L. Coburn and A. Korányi $[\mathbf{1}, \mathbf{2}, 7]$ for domains of rank $\leqslant 2$.

\section{Lemma 3.1. The Toeplitz $C^{*}$-algebra $\mathcal{T}$ acts irreducibly on $H^{2}(S)$.}

Proof. Put $H:=H^{2}(S)$ and let $\pi \in \mathcal{L}(H)$ be a projection commuting with $\mathcal{T}$. Define $f:=\pi(1) \in H$. Then $\pi(g)=\pi\left(T_{g} 1\right)=T_{g} f=g f$ for all $g \in \mathscr{P}(Z)$. Given $z \in D$, define $\varsigma_{z} \in H$ by $\varsigma_{z}(v):=\S(v, z)$ for all $v \in S$, $\delta$ denoting the Szegö kernel (cf. [17, p. 344]). It follows that

$$
\begin{aligned}
\left(\pi\left(\varsigma_{z}\right) \mid g\right)_{S} & =\left(\varsigma_{z} \mid \pi(g)\right)_{S}=\left(\varsigma_{z} \mid g f\right)_{S}=g(z) f(z) \\
& =f(z)\left(\varsigma_{z} \mid g\right)_{S}
\end{aligned}
$$

using the reproducing property of $\delta$. Since $g \in \mathscr{P}(Z)$ is arbitrary, $\overline{f(z)}$ is an eigenvalue of $\pi$, hence $f=0$ or $f=1$ since $D$ is a domain. Q.E.D.

For irreducible domains of tube type there exists a tensor product representation (cf. \$1)

$$
H^{2}(S)=\overline{\mathcal{H}(Z)} \otimes H^{2}(\mathbf{T}) .
$$

Let $\bigcup$ denote the unital $C^{*}$-algebra (acting on $\overline{\mathcal{H}(Z)}$ ) generated by all operators $A_{v}$ for $v \in Z$. Then also $B_{v} \in \mathcal{C}$ and for fixed $t \in \mathbf{T}$, the operators $A_{v}+t B_{v}$ for $v \in Z$ generate an abelian $C^{*}$-subalgebra of $\mathcal{C}$, as follows from Proposition 1.8. In particular, $C$ is generated by two abelian $C^{*}$-subalgebras (cf. [23]).

Proposition 3.2. Let $Z$ be an irreducible JB*-algebra. Then $\mathcal{T}$ is a $C^{*}$-subalgebra of the $C^{*}$-algebra tensor product $\odot \otimes \sigma_{\mathbf{T}}$ (with respect to (3.1)) and the closed commutator ideal $\mathcal{T}^{\prime}$ of $\mathcal{T}$ satisfies $\mathcal{T}^{\prime}=\mathcal{C} \otimes \mathcal{K}_{\mathbf{T}}$. Here $\mathcal{T}_{\mathbf{T}}$ (resp. $\mathcal{K}_{\mathbf{T}}$ ) denotes the Toeplitz $C^{*}$-algebra (resp. the $C^{*}$-algebra of all compact operators) on $H^{2}(\mathbf{T})$.

Proof. The first statement is immediate from Proposition 1.7. Denote by $\xi$ the identity function on $\mathbf{T}$. Then $P:=\left[T_{\xi}^{*}, T_{\xi}\right]=\mathrm{id}-T_{\xi} T_{\xi}^{*}$ is the orthogonal projection from $H^{2}(\mathrm{~T})$ onto $\mathrm{C} \cdot 1$, hence $P \in \mathcal{K}_{\mathrm{T}}$. By Proposition 1.7 the operators id $\otimes P=$ $\left[T_{N}^{*}, T_{N}\right]$ and $A_{v}^{*} \otimes P=\left[T_{N}^{*}, T_{N}\right] T_{l}$ belong to $\mathcal{T}^{\prime}$, where $v \in Z$ and $l:=v^{*}$. Hence $\varrho \otimes \mathcal{K}_{\mathbf{T}} \subset \mathcal{T}^{\prime}$ since $\mathcal{K}_{\mathbf{T}}$ is a simple $C^{*}$-algebra. Conversely, the commutation rules

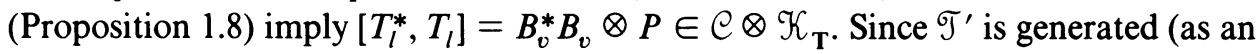
ideal) by such commutators, the assertion follows with Proposition 1.7. Q.E.D.

By [20, Theorem 6.3], the "boundary components" of a bounded symmetric domain $D$ are related to the tripotents of the associated $J B^{*}$-triple $Z$. It will now be shown that to each tripotent $e \in Z$ there corresponds an irreducible representation of 
the Toeplitz $C^{*}$-algebra $\mathcal{T}$. Let $Z=Z_{1}(e) \oplus Z_{1 / 2}(e) \oplus Z_{0}(e)$ be the Peirce decomposition induced by $e$ [20, Theorem 3.13], where $Z_{j}(e):=\left\{z \in Z:\left\{e e^{*} z\right\}=j z\right\}$. Let $S_{e}$ be the Shilov boundary of the bounded symmetric domain $D \cap Z_{e}$ contained in the $J B^{*}$-subtriple $Z_{e}:=Z_{0}(e)$ of $Z$. Endow $S_{e}$ with the probability measure $\mu_{e}$ invariant under $K_{e}:=\operatorname{Aut}\left(Z_{e}\right)^{0}$. Then $e \oplus S_{e} \subset S$, since $S$ consists of all tripotents of maximal rank [20, Theorem 6.5]. Given $f \in \mathcal{C}(S)$, define $f_{e} \in \mathcal{C}\left(S_{e}\right)$ by $f_{e}(w):=f(e+w)$ for all $w \in S_{e}$. Denoting the generic trace of $Z$ by $(\mid)$ put $H_{e}(z):=\exp (z \mid e)$ and define "peaking functions"

$$
h_{e}^{i}(z):=H_{e}^{i}(z) /\left\|H_{e}^{i}\right\|_{S}
$$

for all $i \in \mathbf{N},\|\|_{S}$ denoting the norm in $H^{2}(S)$. For sequences $\left(f_{i}\right)$ and $\left(g_{i}\right)$ in $H^{2}(S)$, we shall write $f_{i} \sim g_{i}$ if $\lim _{i \rightarrow \infty}\left\|f_{i}-g_{i}\right\|_{S}=0$.

Lemma 3.3. Let $Z$ be a $J B^{*}$-triple and $e \in Z$ a tripotent. Then for any $f \in \mathcal{C}(S)$ :

$$
\mu_{e}\left(f_{e}\right)=\lim _{i \rightarrow \infty} \int_{S} f\left|h_{e}^{i}\right|^{2} d \mu .
$$

Proof. Suppose $\sup \left|f_{e}\left(S_{e}\right)\right| \leqslant \delta<\varepsilon$. According to [20, Lemma 6.2], there exist neighborhoods $V \subset U \subset S$ of $e \oplus S_{e}$ such that $\sup |f(U)| \leqslant \varepsilon$ and

$$
\sup _{z \notin U}\left|H_{e}(z)\right|<\inf _{z \in V}\left|H_{e}(z)\right| \text {. }
$$

For the probability measures $\mu_{i}:=\left|h_{e}^{i}\right|^{2} \cdot \mu$ this implies $\lim _{i \rightarrow \infty} \mu_{i}\left(1_{S \backslash U} f\right)=0$ and, hence, letting $\varepsilon \rightarrow \delta$,

$$
\lim _{i \rightarrow \infty} \sup \left|\mu_{i}(f)\right| \leqslant \delta .
$$

It follows that every accumulation point $\lambda$ of $\left(\mu_{i}\right)_{i \in N}$ actually defines a probability measure on $S_{e}$, which is obviously $K_{e}$-invariant since any $\gamma \in K_{e}$ has an extension $g \in K$ fixing $e$. By the uniqueness of $\mu_{e}$, a compactness argument implies $\lim _{i \rightarrow \infty} \mu_{i}(f)=\mu_{e}\left(f_{e}\right)$. Q.E.D.

Corollary. 3.4. For all $p \in \mathscr{P}(Z)$ and $q \in \mathscr{P}\left(Z_{e}\right) \subset \mathscr{P}(Z)$ (via the Peirce projection) we have $T_{p}\left(h_{e}^{i} q\right) \sim h_{e}^{i}\left(T_{p_{e}} q\right)$.

Proof. Since $p-p_{e}$ vanishes on $e \oplus S_{e}$, Lemma 3.3 yields the assertion. Q.E.D.

In order to prove a similar result for the adjoint operators, some preparation is needed. In the next two lemmas, $Z$ is assumed to be irreducible. There exists a frame $\left\{e_{1}, \ldots, e_{r}\right\}$ of $Z$ such that $e=e_{r+1-k}+\cdots+e_{r}$ for some $k \in\{0, \ldots, r\}$. For any fixed $\mu \in \mathbf{N}_{+}^{r-k}$, let $P_{\mu}$ denote the orthogonal projection of $H^{2}(S)$ onto the Hilbert sum of all $K$-modules $E_{m}$ with signatures of the form $m=\left(m_{1}, \ldots, m_{k}, \mu\right)$.

LEMMA 3.5. For $\mu=\left(m_{k+1}, \ldots, m_{r}\right) \in \mathbf{N}_{+}^{r-k}$ put $m:=\left(m_{k+1}, \ldots, m_{k+1}, \mu\right) \in \mathbf{N}_{+}^{r}$. Then

$$
\begin{gathered}
p N_{m} \in P_{\mu}\left(H^{2}(S)\right), \\
T_{l}^{*}\left(p N_{m}\right)=\sum_{k<j \leqslant r}\left(m_{j}+\frac{s}{2}(r-j)+t\right)^{-1} P_{\mu-\varepsilon_{j}}\left(p\left(v \frac{\partial}{\partial z} N_{m}\right)\right)
\end{gathered}
$$

whenever $p \in \mathcal{P}\left(Z_{1}(e)\right), v \in Z_{e}$ and $l:=v^{*}$. 
Proof. Every $\gamma$ in the commutator group of $\operatorname{Aut}\left(Z_{1}(e)\right)^{0}$ has an extension $g \in K$ satisfying $N_{m} \circ g=N_{m}$ and $g \mid Z_{e}=$ id. Hence we may assume $p=N_{1}^{l_{1}} \cdots N_{k}^{l_{k}}$ and, therefore, $p N_{m}=N_{n}$ for some signature $n=\left(n_{1}, \ldots, n_{k}, \mu\right) \in \mathbf{N}_{+}^{r}$. Then (3.2) is clear. Since $v \in Z_{e}$, Lemma 2.9 and Theorem 2.11 imply

$$
T_{l}^{*}\left(p N_{m}\right)=\sum_{k<j \leqslant r}\left(m_{j}+\frac{s}{2}(r-j)+t\right)^{-1}\left(v \frac{\partial}{\partial z}\left(p N_{m}\right)\right)_{n-\varepsilon_{j}} \text { Q.E.D. }
$$

Lemma 3.6. Suppose $q \in \mathfrak{P}\left(Z_{e}\right)$ and $\nu \in \mathbf{N}_{+}^{r-k}$. Then

$$
P_{\nu}\left(h_{e}^{i} q\right) \sim h_{e}^{i} q_{\nu} \text {. }
$$

Proof. We may assume $q$ is the conical polynomial on $Z_{e}$ with signature $\mu \in \mathbf{N}_{+}^{r-k}$. Since $N_{m}-q$ vanishes on $e \oplus S_{e}$, Lemma 3.3 implies $P_{\nu}\left(h_{e}^{i} q\right) \sim P_{\nu}\left(h_{e}^{i} N_{m}\right)$. If $\mu \neq \nu$, then $P_{\nu}\left(h_{e}^{i} N_{m}\right)=0$ by (3.2) and $q_{\nu}=0$. If $\mu=\nu$, then (3.2) implies

$$
P_{\nu}\left(h_{e}^{i} N_{m}\right)=h_{e}^{i} N_{m} \sim h_{e}^{i} q=h_{e}^{i} q_{\nu} \text {. Q.E.D. }
$$

Lemma 3.7. Let $Z$ be a JB*-triple, $p \in \mathscr{P}(Z)$ and $q \in \mathscr{P}\left(Z_{e}\right)$. Then $T_{p}^{*}\left(h_{e}^{i} q\right) \sim$ $h_{e}^{i}\left(T_{p_{c}}^{*} q\right)$.

ProOF. By simple tensor product considerations, we may assume $Z$ is irreducible. Further, using multiplicative properties, we may assume $p=v^{*}$ is linear. If $v \in Z_{e}^{\perp}$, then $p-p_{e}$ vanishes on $e \oplus S_{e}$ and $p_{e}$ is constant, hence applying the Szegö projection $\pi$ and Lemma 3.3 yields the assertion. Now assume $v \in Z_{e}$. Since every $\gamma \in K_{e}$ has an extension $g \in K$ fixing $e$, we may assume $q$ is the conical polynomial on $Z_{e}$ with signature $\mu=\left(m_{k+1}, \ldots, m_{r}\right) \in \mathbf{N}_{+}^{r-k}$. Since $Z_{e}$ is of type $(r-k, s, t)$ and $r-j=(r-k)-(j-k)$ for $j>k$, Theorem 2.12 implies

$$
T_{p_{c}}^{*} q=\sum_{k<j \leqslant r}\left(m_{j}+\frac{s}{2}(r-j)+t\right)^{-1}\left(v \frac{\partial}{\partial z} q\right)_{\mu-\varepsilon_{j}} .
$$

Since $v(\partial / \partial z)\left(N_{m}-q\right)$ vanishes on $e \oplus S_{e}$, Lemmas 3.3 and 3.6 imply

$$
P_{\mu-\varepsilon_{,}}\left(h_{e}^{i}\left(v \frac{\partial}{\partial z} N_{m}\right)\right) \sim P_{\mu-\varepsilon_{,}}\left(h_{e}^{i}\left(v \frac{\partial}{\partial z} q\right)\right) \sim h_{e}^{i}\left(v \frac{\partial}{\partial z} q\right)_{\mu-\varepsilon_{j}} .
$$

Combining this with (3.3) and (3.4), we obtain

$$
T_{p}^{*}\left(h_{e}^{i} q\right) \sim T_{p}^{*}\left(h_{e}^{i} N_{m}\right) \sim h_{e}^{i}\left(T_{p_{e}}^{*} q\right) \text {. Q.E.D. }
$$

Denote by $\mathcal{T}_{0}$ the $*$-algebra generated by all Toeplitz operators $T_{p}$ for $p \in \mathcal{P}(Z)$.

THEOREM 3.8. For each tripotent e of a JB*-triple $Z$ there exists an irreducible representation ("e-symbol") $\sigma_{e}$ of the Toeplitz $C^{*}$-algebra. $\sigma$ on the Hardy space $H^{2}\left(S_{e}\right)$, uniquely determined by one of the following properties:

(i) $\sigma_{e}\left(T_{f}\right)=T_{f_{e}}$ for all $f \in \mathcal{C}(S)$;

(ii) $\lim _{i \rightarrow \infty}\left\|A\left(h_{e}^{i} q\right)-h_{e}^{i}\left(\sigma_{e}(A) q\right)\right\|_{S}=0$, if $A \in \mathcal{T}_{0}$ and $q \in \mathcal{P}\left(Z_{e}\right) \subset \mathcal{P}(Z)$.

Proof. Let $\mathscr{Q}$ denote the set of all operators $A \in \sigma_{0}$ such that (ii) is true for some operator $\sigma_{e}(A)$ on $\mathscr{P}\left(Z_{e}\right)$. By Lemma 3.3, $\sigma_{e}(A)$ is uniquely determined by $A$ and

$$
\left\|\sigma_{e}(A)\right\| \leqslant\|A\|
$$


for the respective operator norms. By definition $\mathcal{Q}$ is an algebra and $\sigma_{e}$ : $\mathbb{Q} \rightarrow$ $\mathcal{L}\left(H^{2}\left(S_{e}\right)\right)$ is a homomorphism. For every $p \in \mathcal{P}(Z)$, it follows from Corollary 3.4 that $T_{p} \in \mathbb{Q}$ and $\sigma_{e}\left(T_{p}\right)=T_{p_{e}}$. Further, Lemma 3.7 implies $T_{p}^{*} \in \mathbb{Q}$ and $\sigma_{e}\left(T_{p}^{*}\right)=T_{p_{e}}^{*}$. Hence $\mathbb{Q}=\mathcal{T}_{0}$ and, by (3.5), $\sigma_{e}$ has a unique extension to a $C^{*}$-algebra homomorphism on $\mathcal{T}$ satisfying (i). Since $\sigma_{e}(\mathcal{T})$ is the Toeplitz $C^{*}$-algebra on $S_{e}, \sigma_{e}$ is irreducible by Lemma 3.1. Q.E.D.

Proposition 3.9. The representations $\sigma_{e}$ of $\mathcal{T}$ for tripotents $e \in Z$ are mutually inequivalent.

Proof. Suppose $e$ and $c$ are different tripotents of rank $k$ and $j$, respectively. Then $e \oplus S_{e} \neq c \oplus S_{c}$. This is clear if $k \neq j$ and it follows from [20, Lemma 6.2] if $k=j$. By Urysohn's theorem there exists $f \in \mathcal{C}(S)$ vanishing on $e \oplus S_{e}$ but not on $c \oplus S_{c}$. Hence $T_{f} \in \operatorname{ker}\left(\sigma_{e}\right) \backslash \operatorname{ker}\left(\sigma_{c}\right)$. Q.E.D.

The irreducible representations corresponding to maximal tripotents $e \in S$ are 1-dimensional, i.e. characters. We will now show that these are the only characters of \%.

Lemma 3.10. Let $\mathcal{T}^{\prime}$ denote the closed commutator ideal of $\mathcal{T}$. Then $T_{f} T_{g}-T_{f g} \in \mathcal{T}^{\prime}$ whenever $f, g \in \mathcal{C}(S)$.

Proof. By Stone-Weierstrass the linear space generated by all functions $\bar{p} q$ for $p, q \in \mathcal{P}(Z)$ is a dense *-subalgebra of $\mathcal{C}(S)$. Hence the assertion follows from

$$
T_{\bar{p} q} T_{\overline{f g}}^{-}-T_{\bar{p} q \overline{f g}}=T_{p}^{*}\left[T_{q}, T_{f}^{*}\right] T_{g} \in \mathcal{T}^{\prime} \text {. Q.E.D. }
$$

Proposition 3.11. The mapping $f \mapsto T_{f}$ induces a $C^{*}$-algebra isomorphism $\mathrm{C}(S) \approx$ $\sigma / \mathcal{T}^{\prime}$. Hence every operator $A \in \mathcal{T}$ has a unique representation $A=T_{f}+A^{\prime}$, where $f \in \mathcal{C}(S)$ and $A^{\prime} \in \mathcal{T}^{\prime}$.

Proof. By Lemma 3.10 the mapping $f \mapsto T_{f}$ is a homomorphism onto the $C^{*}$-algebra $\mathcal{T} / \mathcal{T}^{\prime}$. Since $H^{2}\left(S_{e}\right) \approx \mathbf{C}$ for all $e \in S$, the homomorphisms $\sigma_{e}$ for all $e \in S$ yield a homomorphism $\mathcal{T} \rightarrow C(S)$ vanishing on $\mathcal{T}^{\prime}$. Obviously these homomorphisms are reciprocal. Q.E.D.

Generalizing Proposition 3.11, we can show that the representations $\sigma_{e}$ induced by tripotents $e \in Z$ constitute all irreducible representations of $\mathcal{T}$. This implies, by general $C^{*}$-algebra theory [8, Chapter 10], that $\mathcal{T}$ is a solvable $C^{*}$-algebra of length $r:=\operatorname{rank}(D)\left(\mathrm{cf}\right.$. [10]) with spectral components $S_{k}:=\{e \in Z$ : e tripotent of rank $k$ \} for $0 \leqslant k \leqslant r$. The key fact is that an operator $A \in \mathcal{J}$ satisfying $\sigma_{e}(A)=0$ for all nonzero tripotents $e$ is actually compact. The proof of this is based on the explicit construction of completely positive "smooth" cross-sections $\tau_{k}$ of the $k$-symbol homomorphisms $\sigma_{k}:=\left(\sigma_{e}\right)_{e \in S_{k}}$ for $0 \leqslant k \leqslant r$, generalizing the Toeplitz map $f \mapsto T_{f}$ (for $k=r$ ) and also the "Friedrichs map" for pseudo-differential operators on spheres. The details will appear in a forthcoming publication.

\section{REFERENCES}

1. C. A. Berger and L. A. Coburn, Wiener-Hopf operators on $U_{2}$, Integral Equations Operator Theory 2 (1979), 139-173.

2. C. A. Berger, L. A. Coburn and A. Korányi, Opérateurs de Wiener-Hopf sur les sphères de Lie, C. R. Acad. Sci. Paris Sér. A-B 290 (1980), 989-991. 
3. L. Boutet de Monvel and V. Guillemin. The spectral theory of Toeplitz operators, Ann. of Math. Studies, no. 99, Princeton Univ. Press, Princeton, N. J., 1981.

4. L. Boutet de Monvel, On the index of Toeplitz operators of several complex variables, Invent. Math. 50 (1979), 249-272.

5. H. Braun and M. Koecher, Jordan-Algebren, Springer-Verlag, Berlin and New York, 1966.

6. R. Braun, W. Kaup and H. Upmeier, A holomorphic characterization of Jordan $C^{*}$-algebras, Math. Z. 161 (1978), 277-290.

7. L. A. Coburn, Singular integral operators and Toeplitz operators on odd spheres, Indiana Univ. Math. J. 23 (1973), 433-439.

8. J. Dixmier, $C^{*}$-algebras, North-Holland, Amsterdam, 1977.

9. R. G. Douglas, Banach algebra techniques in operator theory, Academic Press, New York, 1972.

10. A. Dynin, Inversion problem for singular integral operators: $C^{*}$-approach. Proc. Nat. Acad. Sci. U.S.A. 75 (1978), 4668-4670.

11. S. Helgason, Differential geometry and s!mmetric spaces, Academic Press, New York, 1962.

12. L. K. Hua, Harmonic analysis of functions of several complex variables in the classical domains, Transl. Math. Monos., vol. 6, Amer. Math. Soc., Providence, R.I., 1963.

13. J. Janas, Toeplitz operators related to certain domains in $\mathbf{C}^{\prime \prime}$. Studia Math. 54 (1975), 73-79.

14. N. P. Jewell and S. G. Krantz, Toeplitz operators and related function algebras on certain pseudoconvex domains, Trans. Amer. Math. Soc. 252 (1979), 297-311.

15. K. D. Johnson, On a ring of invariant polynomials on a hermitian symmetric space, J. Algebra 67 (1980), 72-80.

16. M. Koecher, An elementary approach to bounded symmetric domains, Rice Univ., Houston, 1971.

17. A. Koráanyi, The Poisson integral for generalized half-planes and hounded symmetric domains, Ann. of Math. (2) 82 (1965), 332-350.

18. D. A. Levine, Sistems of singular integral operators on spheres, Trans. Amer. Math. Soc. 144 (1969), 493-522.

19. O. Loos, Jordan pairs, Springer-Verlag, Berlin and New York, 1975.

20. Bounded slmmetric domains and Jordan pairs, Univ. of California. Irvine, 1977.

21. P. S. Muhly and J. N. Renault, $C^{*}$-algebras of multi-variable Wiener-Hopf operators, Trans. Amer. Math. Soc. 274 (1983), 1-44.

22. R. Narasimhan, Several complex variables, Univ. of Chicago, Chicago, 1971.

23. S. C. Power, Commutator ideals and pseudo-differential $C^{*}$-algebras, Quart. J. Math. Oxford Ser. (2) 31 (1980), 467-489.

24. I. Raeburn, On Toeplitz operators associated with strongly pseudo-convex domains, Studia Math. 63 (1979), 253-258.

25. W. Schmid, Die Randwerte holomorpher Funktionen auf hermitesch symmetrischen Räumen, Invent. Math. 9 (1969), 61-80.

26. M. Takeuchi, Polynomial representations associated with symmetric bounded domains, Osaka J. Math. 10 (1973), 441-475.

27. H. Upmeier, Jordan algehras and harmonic analysis on symmetric spaces, (preprint).

28. U. Venugopalkrishna. Fredholm operators associated with strongly pseudoconvex domains, J. Funct. Anal. 9 (1972), 349-373.

29. G. Warner, Harmonic analysis on semi-simple Lie groups. I, II, Springer-Verlag, Berlin and New York, 1972.

Department of Mathematics, University of Pennsylvania, Philadelphia, Pennsylvania 19104 